ANNALES

POLONICI MATHEMATICI

96.1 (2009)

\title{
Symmetry problems 2
}

\author{
by N. S. HoAng and A. G. Ramm (Manhattan, KS)
}

\begin{abstract}
Some symmetry problems are formulated and solved. New simple proofs are given for some symmetry problems studied earlier. One of the results is as follows: if a single-layer potential of a surface, homeomorphic to a sphere, with a constant charge density, is equal to $c /|x|$ for all sufficiently large $|x|$, where $c>0$ is a constant, then the surface is a sphere.
\end{abstract}

1. Introduction. Symmetry problems are of interest both theoretically and in applications.

A well-known, and still unsolved, symmetry problem is the Pompeiu problem (see [3], [4]). It consists in proving the following:

If $D \subset \mathbb{R}^{n}, n \geq 2$, is homeomorphic to a ball, and the boundary $S$ of $D$ is sufficiently smooth $\left(S \in C^{1, \lambda}, \lambda>0\right.$, is sufficient), and if the problem

$$
\left(\nabla^{2}+k^{2}\right) u=0 \quad \text { in } D,\left.\quad u\right|_{S}=c,\left.\quad u_{N}\right|_{S}=0, \quad k^{2}=\text { const }>0,
$$

has a solution, then $S$ is a sphere.

A similar problem (Schiffer's conjecture) is also unsolved:

If the problem

(2) $\left(\nabla^{2}+k^{2}\right) u=0 \quad$ in $D,\left.\quad u\right|_{S}=0,\left.\quad u_{N}\right|_{S}=c \neq 0, \quad k^{2}=$ const $>0$, has a solution, then $S$ is a sphere.

In [5] it is proved that if

$$
\int_{D} \frac{d y}{4 \pi|x-y|}=\frac{c}{|x|}, \quad \forall x \in B_{R}^{\prime}=\left\{x \in \mathbb{R}^{3}:|x|>R\right\}, c=\text { const }>0,
$$

then $D$ is a ball.

Here and below we assume that $D \subset \mathbb{R}^{3}$ is a bounded domain homeomorphic to a ball, with a sufficiently smooth boundary $S$ ( $S$ being Lipschitz suffices), $B_{R}=\{x:|x| \leq R\}$, and $B_{R} \supset D$. By $\mathcal{H}$ we denote the set of all

2000 Mathematics Subject Classification: 35J05, 31B20.

Key words and phrases: symmetry problems, potential theory. 
harmonic functions in a domain which contains $D$. By $|D|$ and $|S|$ we denote the volume of $D$ and the surface area of $S$, respectively.

Our goal is to give a simple proof of the three symmetry-type results formulated in Theorem 1 in Section 2.

In [7] the following result is obtained:

If

$$
\Delta u=1 \quad \text { in } D,\left.\quad u\right|_{S}=0,\left.\quad u_{N}\right|_{S}=\mu=\text { const }>0,
$$

then $S$ is a sphere.

This result is obtained by A. D. Aleksandrov's "moving plane" argument, and is equivalent to the following:

If

$$
\frac{1}{|D|} \int_{D} h(x) d x=\frac{1}{|S|} \int_{S} h(s) d s, \quad \forall h \in \mathcal{H},
$$

then $S$ is a sphere.

The equivalence of (4) and (5) can be proved as follows.

Suppose (4) holds. Multiply (4) by an arbitrary $h \in \mathcal{H}$, integrate by parts and get

$$
\int_{D} h(x) d x=\mu \int_{S} h(s) d s .
$$

If $h=1$ in (6), then one gets $\mu=|D| /|S|$, so (6) is identical to (5).

Suppose (5) holds. Then (6) holds. Let $v$ solve the problem $\Delta v=1$ in $D$, $\left.v\right|_{S}=0$. This $v$ exists and is unique. Using (6), the equation $\Delta h=0$ in $D$, and the Green's formula, one gets

$$
\mu \int_{S} h(s) d s=\int_{D} h(x) d x=\int_{D} h(x) \Delta v d x=\int_{S} h(s) v_{N} d s .
$$

Thus,

$$
\int_{S} h(s)\left[v_{N}-\mu\right] d s=0, \quad \forall h \in \mathcal{H} .
$$

The set of restrictions to $S$ of all harmonic functions in $D$ is dense in $L^{2}(S)$ (see, e.g., [5]). Thus, (8) implies $\left.v_{N}\right|_{S}=\mu$. Therefore, (4) holds.

2. Results and proofs. Our main results are formulated in the following theorem:

TheOREM 1. Let $D \subset \mathbb{R}^{3}$ be a bounded domain homeomorphic to a ball, $S$ be its Lipschitz boundary, $D^{\prime}:=\mathbb{R}^{3} \backslash D$. If any one of the following assumptions holds, then $S$ is a sphere: 
1. We have

$$
u(x):=\int_{S} \frac{d s}{4 \pi|x-s|}=\frac{c}{|x|}, \quad \forall x \in B_{R}^{\prime}, \quad c=\text { const },
$$

where $B_{R}^{\prime}:=\{x:|x|>R\}, D \subset B_{R}, B_{R}:=\mathbb{R}^{3} \backslash B_{R}^{\prime}$.

2. We have

$$
\frac{1}{|S|} \int_{S} h(s) d s=h(0), \quad \forall h \in \mathcal{H} .
$$

3. There exists a solution to the problem

$$
\Delta_{y} u=\delta(y) \quad \text { in } D,\left.\quad u\right|_{S}=0,\left.\quad u_{N}\right|_{S}=c_{1}=\text { const, }
$$

where $\delta(y)$ is the delta-function.

In (10), 0 is the origin, $0 \in D,|S|$ is the surface area of $S$, and $\mathcal{H}$ is the set of all harmonic functions in a domain containing $D$.

Proof. 1. Assume (9). Then $c=|S| /(4 \pi)$ as one can see by letting $|x| \rightarrow \infty$. If (9) holds for all $x \in B_{R}^{\prime}$ then, by the unique continuation property for harmonic functions, (9) holds for all $x \in D^{\prime}$. Let $N_{s}$ be the unit normal to $S$ at the point $s \in S$, pointing into $D^{\prime}$. The known jump formula for the normal derivative of a single-layer potential ([2, p. 14]) yields

$$
u_{N_{s_{0}}}^{+}=u_{N_{s_{0}}}^{-}+1, \quad u_{N_{s_{0}}}^{-}=-\frac{|S|}{4 \pi} \frac{N_{s_{0}} \cdot s_{0}}{\left|s_{0}\right|^{3}}, \quad s_{0} \in S .
$$

If $S$ is not a sphere, then there exists an $s_{0} \in S$ with $\left|s_{0}\right| \leq|s|$ for $s \in S$. The ball $B_{\left|s_{0}\right|}$ of radius $\left|s_{0}\right|$, centered at the origin, is contained in $D$. At the point $s_{0}$ the normal $N_{s_{0}}$ to $S$ is directed along the vector $s_{0}$, so

$$
u_{N_{s_{0}}}^{-}=-\frac{|S|}{4 \pi\left|s_{0}\right|^{2}}<-1,
$$

because $|S|>4 \pi\left|s_{0}\right|^{2}$ by the isoperimetric inequality ([1]). This and formula (12) imply

$$
u_{N_{s_{0}}}^{+}<0
$$

On the other hand,

$$
u(s)=\frac{1}{4 \pi|s|} \leq \frac{1}{4 \pi\left|s_{0}\right|} .
$$

So the function $u(x)$, harmonic and continuous in $D$, attains its maximum on $S$ at the point $s_{0}$, because $\left.u\right|_{S}=\left.\frac{1}{4 \pi|s|}\right|_{S}$. Therefore, by the maximum principle,

$$
u(x) \leq u\left(s_{0}\right), \quad \forall x \in D .
$$


In particular, $u\left(s_{0}\right)-u\left(s_{0}-\epsilon N_{s_{0}}\right) \geq 0$ for all sufficiently small $\epsilon>0$. Consequently, $u_{N_{s_{0}}} \geq 0$. This contradicts (14), and the contradiction proves that $S$ is a sphere.

2. Assume (10). Let $h(y)=\frac{1}{4 \pi|x-y|}, x \in D^{\prime}, y \in D$. This function is harmonic in $D$. Thus, (10) yields (9):

$$
\int_{S} \frac{d s}{4 \pi|x-s|}=\frac{|S|}{4 \pi|x|}=\frac{c}{|x|}, \quad \forall x \in D^{\prime}, \quad c:=\frac{|S|}{4 \pi} .
$$

We have already proved that (16) implies that $S$ is a sphere.

3. Assume (11). Multiply (11) by $1 /(4 \pi|x-y|), x \in D^{\prime}$, integrate over $D$, and then integrate by parts to get

$$
c_{1} \int_{S} \frac{d s}{4 \pi|x-s|}=\frac{1}{4 \pi|x|}, \quad \forall x \in D^{\prime} .
$$

By the result proved in assertion 1, this implies that $S$ is a sphere.

\section{References}

[1] H. Federer, Geometric Measure Theory, Springer, Berlin, 1969.

[2] A. G. Ramm, Scattering by Obstacles, Reidel, Dordrecht, 1986.

[3] —, The Pompeiu problem, Appl. Anal. 64 (1997), 19-26.

[4] -, Necessary and sufficient condition for a domain, which fails to have Pompeiu property, to be a ball, J. Inverse Ill-Posed Probl. 6 (1998), 165-171.

[5] -, A symmetry problem, Ann. Polon. Math. 92 (2007), 49-54.

[6] A. G. Ramm and E. Shifrin, Symmetry problems in the elasticity theory problem for plane cracks of normal rapture, Prikl. Mat. Mekh. 69 (2005), 135-143 (in Russian); English. transl.: J. Appl. Math. Mech. 69 (2005), 127-134.

[7] J. Serrin, A symmetry problem in potential theory, Arch. Ration. Mech. Anal. 43 (1971), 304-318.

Mathematics Department

Kansas State University

Manhattan, KS 66506-2602, U.S.A.

E-mail: nguyenhs@math.ksu.edu ramm@math.ksu.edu

Received 31.10.2008

and in final form 28.1.2009 